\title{
The Influence of Psychological Safety and Personality on Technology Acceptance of Team-Based Technology in Global Virtual Teams
}

\author{
Peter W. Cardon \\ University of Southern California \\ cardon@marshall.usc.edu \\ Haibing Ma \\ LeapIn Information \\ Technology(Shanghai) Co., LTD \\ martin.ma@leapin.io
}

\author{
Carolin Fleischmann \\ Rosenheim Technical University of \\ Applied Sciences \\ carolin.fleischmann@th-rosenheim.de \\ Ann Springer \\ Brigham Young University- Hawaii \\ ann.springer@byuh.edu
}

\author{
Jolanta Aritz \\ University of Southern California \\ aritz@marshall.usc.edu \\ Scott Springer \\ Brigham Young University- \\ Hawaii \\ scott.springer@byuh.edu
}

\begin{abstract}
Collaboration platforms for teams, such as Slack, are increasingly used in virtual teams. Conventional wisdom suggests attitudes about adopting these types of platforms is primarily driven by their affordances. Our project emerged from the premise that psychological safety and personality traits can also significantly influence attitudes related to technology adoption. This research of roughly 300 global virtual teams showed that psychological safety influences views of collaboration platforms in terms of performance expectancy, effort expectancy, and hedonic motivation. In addition, this research showed that personality traits influence views of collaboration platforms. These findings about psychological safety and personality traits suggest a team-development approach is an integral component of the technology adoption process. Recommendations for future research are provided.
\end{abstract}

\section{Introduction}

Collaboration platforms for teams, such as Slack and Microsoft Teams, have dramatically grown in use over the past few years. Daily users on Slack rose from 6 million to 12 million between the end of 2019 and 2020 [1]. Similarly, Microsoft Teams grew from 50 million daily users to 145 million users between 2019 and 2020 [2]. The transition to more team communication and collaboration occurring on these types of collaboration platforms is not surprising. Many scholars have predicted that collaboration platforms (referred to in many ways over the years, including enterprise social networks, enterprise social systems, internal social media, intranet 2.0) would replace email as the primary form of digital business communication [3].

Yet, many scholars predicted this transition would occur much more rapidly. They certainly did not anticipate a global pandemic would accelerate the adoption of these tools. Most of the early proponents of online collaboration systems adopted a technological affordances view. They assumed that because collaboration platforms provided interactive and collaborative features that in some ways mimicked social media, professionals would naturally recognize the technical superiority of these tools compared to email, particularly for teamwork. Proponents of these platforms expected widespread and rapid adoption in teams $[3,4,5,6,7]$. In practice, adoption has been slower. This naturally begs the question: if superior technological affordances do not drive rapid adoption, what does? In part, our study seeks to address this question in the context of Slack use. We start from the premise that psychological safety is fundamental to driving positive views of a team-based technology. Further, we examine the degree to which personality influences views of team-based technology. One premise of our study is that psychological safety and personality influence key constructs of technology acceptance, including performance expectancy (PE), effort expectancy (EE), and hedonic motivation (HED).

\section{Theoretical background}

Global virtual teams (GVT) face collaboration challenges that are less frequently encountered by colocated teams. They are geographically dispersed and communicate via technology. Both these defining factors mean that they are missing many social and emotional cues that are present in face-to-face 
communication. This is accentuated by the fact that GVT are often multicultural where misunderstandings are more frequent and are affected by different languages, mindsets, and cultures. All this leads to difficulties in creating trust and accountability in virtual teams $[8,9]$.

This study examines PE, EE, and HED in the context of GVTs that use a team collaboration platform, Slack. We first review the nature of collaboration platforms, their affordances, and technology acceptance. Then, we propose how psychological safety, personality, and several additional factors affect $\mathrm{PE}, \mathrm{EE}$, and HED in the context of team collaboration platforms.

\subsection{Collaboration platforms and their affordances}

Collaboration platforms (also referred to as team communication platforms and team messaging platforms) provide a suite of tools for teams to communicate and collaborate. These tools include but certainly are not limited to messaging, file sharing, coediting, calendaring, project management, and video meetings. Tags, mentions, channels, and notifications are just a few of the features that allow teams to ensure the right people are involved at the right moments in idea generation, content development, and decision making. Collectively, this communication visibility affords collaborative workflows, multicommunication, and attention allocation [9].

We specifically explore the team collaboration platform Slack. Slack was designed to bring a social media type experience to the workplace and caters to the messaging culture of Millennials and Gen Z. It allows easy access to employees across organizations and emphasizes friendly and warm communication (i.e., bright default colors, encouragement to use emoticons and other visual messages, prominently displayed profile pics) $[10,11]$.

\subsection{Technology acceptance}

To investigate the acceptance of Slack, we base our research on the UTAUT models [12,13], which are among the most prominent and widely used technology acceptance models. In UTAUT, intention to use a technology is influenced by several factors, such as performance expectancy, effort expectancy (UTAUT), and hedonic motivation (UTAUT2).

In UTAUT2, performance expectancy, effort expectancy, social influence, facilitating conditions, hedonic motivation, price value, and habit influence behavior intention [13]. These factors need to be adjusted to account for the set-up of our study. We excluded social influence because of the involuntariness of using the technology; facilitating conditions because every participant in the study had the same resources and support to use the technology; and price value because the tool was free of charge for study participants. UTAUT2 was specifically developed to measure acceptance of consumer technology [13]. Therefore, we also drew on the original UTAUT model to account for the organizational context of our study.

We focused on individuals' performance expectancy (PE), effort expectancy (EE), and hedonic motivation (HED). Past studies found that these variables have been significant predictors of intention to use a technology $[14,15,16]$. PE, in particular, has a strong influence on behavior intention [12,13]. PE and EE represent extrinsic motivation. While $\mathrm{PE}$ is an outcome-oriented factor, EE measures the ease of use of the technology and whether a user believes that he or she can independently and easily navigate the technology. HED represents the attitudes towards the process of using the technology and measures intrinsic motivation. When it is fun to use a technology, people have a higher intention to use it $[14,17]$.

Some recent research has explored potential antecedents to PE, EE, and HED in the context of technology adoption. For example, Chao adapted the UTAUT model with the hypothesis that self-efficacy was an antecedent to HED. This research confirmed this relationship in the context of m-learning [19]. In the context of collaboration technologies (e.g., Slack), some scholars have proposed that a variety of collaborationrelated constructs should serve as antecedents to PE, EE, HED, and other constructs in the UTAUT model [20]

\subsection{Psychological safety and technology acceptance}

Psychological safety is defined as the "shared belief held by members of a team that the team is safe for interpersonal risk taking" [18]. It includes an assessment of the team environment as to how others will react to seeking feedback, pointing out mistakes, or pitching ideas [18]. If the team environment is perceived as nonthreatening and no negative consequences are expected when expressing oneself, a person feels psychologically safe [19]. While the concept is related to cohesion and trust, it is different: In contrast to cohesion, it implies confidence to disagree rather than group think. Trust is other-oriented ("Can I trust you?"); whereas psychological safety is self-oriented ("How will others respond to my behavior?") [18].

Abundant research shows that psychological safety is integral to team processes and performance [20]. Similar results have been found for virtual environments (e.g. $[19,21,22,23])$, but studies on psychological safety 
in virtual teams that never meet in person are scarce $[24,25,26]$. It's likely that psychological safety is even more important for virtual teams. Gibson and Gibbs found that virtuality of collaboration has negative effects of innovation, but this negative effect can be mitigated by psychological safety [21]. Zhang et al. found that psychological safety increases the intention to continue sharing knowledge in virtual communities [19]. Kirkman et al. found that psychological safety increases team performance, particularly for teams with high national diversity. National diversity is the norm in the GVT in the sample of this study. Likewise, Kirkman et al. found that rich communication media increases team performance in highly diverse teams [27]. To foster psychological safety when team members never meet in person, scholars note the importance of accepting virtual team challenges, connecting as people, and agreeing on ground rules [25].

Some research has explored the premise that psychological safety or other measures of team dynamics influence technology adoption and use. One study showed that student teams were more likely to perceive groupware as useful and easy to use based on the psychological safety established within the teams [28]. Further, some researchers suggest that with the massive disruptions of COVID-19 in terms of emotional distress and use of new technologies, psychological safety is more important in online environments than in the past [29]. Another study showed that psychological safety allowed online communities to perform more effectively [30].

Research about the use of Slack in GVT suggests that psychological safety may be an important foundation from which effective Slack use emerges. For example, a study of teams of Chinese and European engineering teams found that a variety of barriers included the low availability of key people, the absence of support for unscheduled meetings, and unbalanced activity among team members. Team members rarely held unscheduled meetings and rarely discussed expectations about how to use Slack [31]. A study of GVT located in the United States and Mexico showed how activity on Slack tended to mirror the real-life interactions. In other words, the real-time interactions via in-person and online conferencing and related psychological safety preceded similar clusters of activity on Slack [32]. Particularly team-based technologies such as Slack, we consider psychological safety as a building block for successful adoption. Regardless of the affordances offered by the technologies, team members are likely hesitant to maximize the use of these affordances unless they are comfortable sharing their ideas with one another and do not fear negative consequences for unpopular views. Thus, it's likely that team members will view Slack and its affordances positively only if they feel psychological safety to open up with their teammates via Slack.

Critics of technology acceptance models suggest these models rely too heavily on individual decisionmaking frameworks and do not adequately capture the influence of group and social dynamics. While the UTAUT model has incorporated social influence into the model, it is treated "in the limited senses of either a constraint or force on the decision maker and perceived as originating from 'other people whose opinions are important to me." A more significant role for social dynamics is needed to address technology adoption when more than one user is necessary for the use of technology [35]. Given the team-based nature of Slack, we adopt the view that psychological safety serves as a fundamental consideration in views of Slack. Thus, we propose the following:

H1: Psychological safety leads to more positive views of $\mathrm{PE}, \mathrm{EE}$, and $\mathrm{HED}$ in collaboration platforms (i.e., Slack).

\subsection{Personality and technology acceptance}

Personality was not proposed as a direct influence on technology acceptance in the Technology Acceptance Model (TAM) and UTAUT models [33]. In the past 10 to 15 years, some studies have explored how personality traits influence technology acceptance, particularly related to performance expectancy and effort expectancy $[33,34,35,36]$, yet the relationships between personality traits and technology acceptance remains relatively unexplored in the context of team collaboration platforms.

Our study employed the NERIS $\AA$ model of personality that is adapted from the trait personality model of Myers-Briggs [37]. The personality traits include introversion versus extroversion, observant versus intuitive, thinking versus feeling, and judging versus prospecting. Recent research has demonstrated the validity of this personality assessment [38]. In addition to theoretical reasons, we adopted the NERIS ${ }^{\circledR}$ model for several practical reasons. It is an easy-to-use online personality assessment that is free to any person. It provides a variety of online resources to help users interpret their own results. Importantly, it provides publicly available country-by-country information, which is particularly helpful in the context of global virtual teams.

We formed several hypotheses related to performance expectancy (PE). As far as effort expectancy, we did not anticipate any significant differences, particularly given past studies that reveal Slack is considered natural and easy to use by Millennials. Likewise, we did not anticipate any 
differences based on hedonic motivation (other than a hypothesis about extroversion). Introverted individuals tend to prefer solitary activities, lose energy from social interaction, and are more sensitive to sensory stimulation. Extroverted individuals tend to prefer group activities, draw energy from social interaction, and display more enthusiasm than introverts. Scholars have proposed that social presence in collaboration technologies is an antecedent of PE [20], and we suggest that this need will be particularly pronounced for extroverts. As a team-based tool that is highly social and interactive in nature, we propose that extroverted individuals will form more positive views of Slack's usefulness (PE) and enjoyability (HED):

H2a: Extroverted personality types will be associated with higher PE.

$H 2 b$ : Extroverted personality types will not be associated with any differences related to EE.

$H 2 c$ : Extroverted personality types will be associated with higher HED.

Observant individuals tend to be practical and pragmatic. They adopt habits based in what has worked in the past. Intuitive individuals tend to be imaginative and curious. They embrace change and do not feel bound by stability. Slack is a tool that allows team members to communicate in novel ways and construct channels for specific collaboration purposes. Thus, we propose intuitive individuals will see more usefulness in the evolving structures within Slack:

$H 2 d$ : Intuitive personality types will be associated with higher $\mathrm{PE}$.

$H 2 e$ : Intuitive personality types will not be associated with any differences related to EE.

$H 2 f$ : Intuitive personality types will not be associated with any differences related to HED.

Thinking individuals tend to focus on objectivity and rationality. They prioritize efficiency over cooperation. Feeling individuals tend to be more emotionally expressive and focus more on social harmony. As a primarily writing-based tool, we propose that thinking individuals are more likely to see value in carefully constructing their thoughts. Thus we propose thinking individuals will see more usefulness in Slack:

$H 2 g$ : Thinking personality types will be associated with higher PE.

$H 2 h$ : Thinking personality will not be associated with any differences related to EE.

$H 2 i$ : Thinking personality will not be associated with any differences related to HED.
Judging individuals tend to be decisive and organized. They value clarity, structure, and planning. Prospecting individuals tend to be flexible, relaxed, and nonconforming. On the one hand, Slack provides structure via channels and other features. Yet, it also explicitly caters to a more flexible and relaxed team environment. Thus, we view it as unlikely that this personality dimension influences the variables in question.

$H 2 j$ : Judging personality types will not be associated with any differences related to PE.

$H 2 k$ : Judging personality types will not be associated with any differences related to EE.

$\mathrm{H} 2 \mathrm{l}$ : Judging personality types will not be associated with any differences related to HED.

\subsection{Prior technology use, time zone challenges, and gender}

Prior experience with a technology is considered predictive of higher technology acceptance in the UTAUT model [13] and specifically in the context of collaboration technologies [20]. Thus, we propose prior Slack use will lead to higher technology acceptance in terms of PE, EE, and HED.

H3a: Prior Slack use leads to higher PE.

$H 3 b$ : Prior Slack use leads to higher EE.

$H 3 c$ : Prior Slack use leads to higher HED.

Gender has been an important element in technology acceptance models for the past twenty years. Generally, men tend to prioritize usefulness whereas women tend to prioritize ease of use [39]. Women have been found to hold more positive views of technology that is fun and playful [40]. Thus, we propose the following:

H4a: Men will hold more positive perceptions of Slack in terms of PE.

$H 4 b$ : Women will hold more positive perceptions of Slack in terms of EE.

$H 4 c$ : Women will hold more positive perceptions of Slack in terms of HED.

Finally, GVT face significant challenges with time zone differences, which likely influence their views of various technologies. One challenge GVT face is that communicating in real time. Slack can be an effective platform for capturing asynchronous communication and collaboration, thus helping team members across time zones continue to work together. Yet, time zone differences can be a source of frustration in coordination and decision making. Thus, we propose the following: 
H5a: Individuals who perceive significant time zone challenges will hold more positive perceptions of Slack in terms of PE.

$H 5 b$ : Individuals who perceive significant time zone challenges will hold more negative perceptions of Slack in terms of EE.

$H 5 c$ : Individuals who perceive significant time zone challenges will hold more negative perceptions of Slack in terms of HED.

\section{Methodology}

\subsection{Sample}

The participants in our study worked in virtual teams and used a combination of Slack and Zoom to work with teammates dispersed around the globe. Team members held weekly meetings on Zoom and used Slack for communication and collaboration between meetings. The six-week-long project required participants to collaborate on a consulting project for one of four US-based Fortune 100 companies. The final deliverable was a written report that included analysis and recommendations.

In the project, undergraduate and MBA students from various disciplines were placed in GVT that included members from different institutions and countries. They never met in person. The data was collected in Spring 2019, Spring 2020, and Fall 2020. Project participants from roughly 20 universities located in eleven societies, including the United States, India, Canada, Lithuania, Finland, Spain, France, Germany, Singapore, Taiwan, and Hong Kong.

A total of 1,814 students in roughly 300 teams participated in the project. Students worked in teams of 5 or 6 members. Participants were asked to complete two quantitative surveys - one at the beginning and one at the end of the project. The surveys included identifiers to match the responses of pre- and postsurveys. They were available in English language to ensure semantic equivalence. All study participants had a working level of English proficiency, which was assessed by formal test scores, professors' ratings, and self-ratings.

1,419 participants completed the pre-project survey (46.2\% male; $53.3 \%$ female, $0.6 \%$ prefer not to say/other). 1,384 participants completed the postproject survey. A total of 1,077 of these students completed both the pre-survey and the post-survey. While the participants studied in one of eleven countries, the survey respondents originally came from 70 countries. The most common countries of origin included the following: United States (806), India (172), China (89), Canada (58), France (41), Lithuania (24),
Spain (19), Germany (16), Mexico (14), Philippines (14), and South Korea (14).

\subsection{Measures}

For our measures of technology acceptance, we adapted items from Venkatesh et al.'s UTAUT models. Due to the specific context of our study, we limited our analysis to PE, EE, and HED. The 4-item-measure of PE was adopted from the original UTAUT model [12] to include an item on the organizational and project context ("Slack increased my team's chances of getting a better grade."). EE and HED were adopted from UTAUT2 [13]. EE has four items, e.g. "Slack was easy to use" (post-survey). HED included three items, e.g. "Using Slack was fun" (post-survey). All items were measured on a 7-point Likert scale from $1=$ completely disagree to 7 = completely agree. Cronbach's alpha for PE was .96., EE was .96, and HED was .94. Table 3 in the Appendix shows these UTAUT items.

Psychological safety was measured using an adapted 8-item scale from Edmondson (1999). Items included "I was afraid of making mistakes" (reverse coded) and "It was easy to ask for help from my team members". The items were measured on a 7-point Likert scale from $1=$ completely disagree to $7=$ completely agree. The Cronbach's alpha for these items was .75. Table 2 in the Appendix presents these items.

All individuals involved in this virtual team project completed a personality test before starting the program. They shared their personalities with their teammates as part of team-building exercises in the first week. Team members took the NERIS $®$ personality model (16 personalities.com) that builds on the trait model of Myers-Briggs but avoids difficult-to-measure cognitive functions. Virtual team members provided their personality traits based on this assessment: introversion versus extroversion, observant versus intuitive, thinking versus feeling, and judging versus prospecting, and assertive versus turbulent. Our sample showed the following breakdowns of personality traits: extroversion: $57.5 \%$, introversion: $42.5 \%$; observant: $40.1 \%$, intuitive: $59.9 \%$; thinking: $39.8 \%$, feeling: $60.2 \%$; judging: $60.5 \%$, prospecting, $39.5 \%$.

\section{Findings}

One of the important goals of this six-week virtual team project is establish psychological safety. This is a challenging proposition given the fact that team members have never met before the project, never meet in person during the project, and each resided in different locations with different institutional cultures. Overall, roughly two thirds of virtual team members felt comfortable speaking up and felt appreciated. About 
half felt invested and belonging. Table 2 in the Appendix provides additional details for these survey items.

Based on the UTAUT items, virtual team members broadly consider Slack easy to use and an important tool to improve performance. Roughly 80 percent felt Slack was easy to use on related survey items, and roughly 60 to 70 percent felt Slack improved their performance. There was less support for how enjoyable Slack was to use. Roughly half thought Slack was interesting or fun. Roughly two thirds thought it was enjoyable. Table 3 in the Appendix provides additional information about the UTAUT items.

Table 1 presents three regression models with PE, EE, and HED as dependent variables. Our primary hypothesis (H1) was that higher psychological safety in virtual teams would influence positive views of a teambased collaboration platform. This hypothesis was confirmed in terms of all measured UTAUT constructs: PE, EE, and HED.

Most hypotheses related to personality traits were supported. Extroverted, intuitive, and thinking personality traits led to higher perceptions of PE, yet judging had no impact on PE. We hypothesized no impacts of personality traits on EE. This was true for extroverted, intuitive, and judging personality traits. However, the thinking personality trait was positive related to EE counter to expectations. We hypothesized that extroverted personality types would be positively related to hedonic motivation, but this was not the case.

As hypothesized, prior use of Slack increased positive perceptions of PE, EE, and HED. Only one of our three hypotheses about time zone differences was confirmed: perceived time zone challenges did lead to lower perceptions of HED. However, contrary to our hypotheses, perceived time zone challenges had no impact on PE or EE. Finally, two of our three hypotheses regarding gender were confirmed. Women perceived EE and HED more positively. However, counter to our hypothesis, men did not perceive Slack as higher in PE.

\section{Discussion}

This study makes several contributions. First, it shows that common UTAUT constructs-PE, EE, and HED - may involve antecedents. Most research about technology adoption in the TAM, UTAUT, and UTAUT2 traditions have not explored predictors of PE, EE, and HED. Yet, scholars have suggested that particularly in the case of collaborative technologies, there may be many predictors of PE, EE, and HED [20]. This study showed this may be the case in the context of GVTs and Slack. Second, it showed that psychological safety influences perceptions of PE, EE, and HED. This broadens the conversation about psychological safety, which has been highly researched in F2F teams but less so in virtual teams. Third, it shows that personality dimensions may influence PE, EE, and HED. Few studies have explored the connections between personality and technology adoption [37] [38], thus the significant findings of this study demonstrate this is an important line of inquiry.

All virtual teams must make decisions about the collaboration platforms and other communication tools they will use. One important approach is to carefully evaluate the affordances of these platforms. In other words, it is important to seek superior technical features that align with team goals and tasks. Yet, relying exclusively on an affordances approach may not rapidly drive adoption. Other factors, such as psychological safety, may significantly impact how positively team members view the technologies they use. Our primary premise for this project was that psychological safety

Table 1. Regression analysis on performance, effort, and attitude.

\begin{tabular}{|c|c|c|c|c|c|c|}
\hline & \multicolumn{2}{|c|}{$\begin{array}{c}\text { Model 1: } \\
\text { Performance Expectancy }\end{array}$} & \multicolumn{2}{|c|}{$\begin{array}{c}\text { Model 2: } \\
\text { Effort Expectancy }\end{array}$} & \multicolumn{2}{|c|}{$\begin{array}{c}\text { Model 3: } \\
\text { Hedonic Motivation }\end{array}$} \\
\hline & $\beta$ & $p$ & $\beta$ & $p$ & $\beta$ & $p$ \\
\hline Psychological safety & .287 & $<.001 * * *$ & .346 & $<.001 * * *$ & .354 & $<.001 * * *$ \\
\hline Time zone challenges & .007 & .826 & -.049 & .093 & -.059 & $.044^{*}$ \\
\hline Prior use of Slack & .159 & $<.001 * * *$ & .154 & $<.001 * * *$ & .118 & $<.001 * * *$ \\
\hline Gender (woman) & .045 & .121 & .078 & $.006^{* *}$ & .083 & $.003 * *$ \\
\hline \multicolumn{7}{|l|}{ Personality traits } \\
\hline Extroverted & .059 & $.043^{*}$ & .018 & .526 & .027 & .341 \\
\hline Intuitive & .077 & $.016^{*}$ & .015 & .635 & .018 & .658 \\
\hline Thinking & .077 & $.029 *$ & .074 & $.032 *$ & .037 & .180 \\
\hline Judging & .015 & .659 & -.016 & .623 & .009 & .616 \\
\hline
\end{tabular}


would positively influence views of a team collaboration platform. This research showed that indeed psychological safety affects positive perceptions of performance expectancy, effort expectancy, and hedonic motivation for Slack.

A lot of research has emphasized the need for psychological safety in co-located, in-person teams and remote, virtual teams. This research adds to the rationale for emphasizing psychological safety in virtual teams: virtual team members who operate entirely via technology will view the technology more positively if they first establish psychological safety. Our research resides in the context of short-term virtual teams, an increasingly common approach to collaboration. In the interest of efficiently meeting impending deadlines, short-term virtual teams may not take the time necessary to establish psychological safety. This research supports the notion that doing so may smoothen the technologymediated environment. Virtual teams, even if they will only function for four to six weeks, should allocate time to build relationships and establish ground rules. Since psychological safety itself influences views of technology and virtual teams often rely entirely on technology-mediated communication, it is essential not to overlook team development.

Our research also suggests personality traits may influence views of technology. In particular, personality dimensions were most influential on PE. A common approach in industry is for teams building team profiles using personality assessment tools. This is an approach we adopt in the first week of the project for teambuilding purposes. Understanding how personality traits impact views of technology may be useful knowledge for virtual team members to help them understand their own reactions to technologies in the virtual environment. This research suggests that individuals with extroverted, intuitive, and thinking personality traits tend to more positively view the usefulness of Slack. These results speak to the importance of building a team and creating psychological safety that is positively correlated with media tools adaption to counter varying degrees of technology acceptance.

\section{Limitations and directions for future research}

Our research benefits from a much larger sample than most studies of virtual teams. Yet, it is limited in several ways. First, it is a student sample. Thus, it is likely more reflective of Gen $\mathrm{Z}$ individuals. We recommend additional studies that explore the influence of psychological safety and personality traits on views of technology with virtual teams of additional generational groups. Second, it is based on the experiences of short-term global virtual teams (in this case, six weeks) with the use of Slack. It would be useful to explore the relationships between psychological safety and personality on technology acceptance in the context of longer-term virtual teams and with other platforms. In the American context, Microsoft Teams and Slack are designed with some similar features yet differ in a variety of ways. For example, Slack tends to emphasize a much more informal and flexible (i.e., wide range of app plug-in choices) approach to team collaboration. One of its defining features is an infrastructure built around channels. Microsoft Teams tends to emphasize more formal approaches to collaboration and coordination built on the Microsoft suite of tools. Of course, there are many types of collaboration platforms across the globe. For example, in China many professionals choose between WeChat and Ding Talk with somewhat similar contrasts. Going forward, we see value in comparative studies of various collaboration platforms.

This research also explored potential antecedents of key factors-PE, EE, and HED - in technology adoption models. While this aligns with theoretical propositions of technology acceptance in collaborative settings by some scholars [20], this is an approach outside of most UTAUT and UTAUT2 research. We recommend future research about Slack and other collaboration platforms that is able to provide multilevel analysis that includes antecedents to the UTAUT constructs, UTAUT constructs, behavioral intentions, and behaviors.

Finally, we note that the importance of psychological safety and trust [9] may continue to grow in importance for virtual settings in upcoming years for several reasons. First, the widespread use of collaboration platforms, such as Slack, during the pandemic has demonstrated to millions of business leaders and employees that virtual teamwork can be at least as productive and efficient as F2F teamwork. Second, the use of collaboration tools, such as Slack, create larger employee digital footprints than ever before. Without psychological safety and trust, employees are more likely to worry about how their data is used by their organizations [45]. Thus, we recommend continued exploration of how psychological safety influences how virtual team members view and use various collaboration platforms.

\section{Conclusion}

Collaboration platforms for teams, such as Slack, are increasingly used in virtual teams. This research of roughly 300 global virtual teams showed that psychological safety influences views of collaboration platforms in terms of performance expectancy, effort expectancy, and hedonic motivation. In addition, this 
research showed that personality traits influence views of collaboration platforms. These findings suggest that PE, EE, HED, and other key factors in technology acceptance models may have antecedents in collaborative technology environments. Additional research is needed to explore the influence of psychological safety on key drivers of technology acceptance. The research highlights the need for managers of virtual teams to focus on psychological safety.

\section{References}

[1] D. Curry, "Slack Revenue and Usage Statistics (2021)," Business of Apps, 6 March 2021. [Online]. Available:

https://www.businessofapps.com/data/slackstatistics/. [Accessed 13 June 2021].

[2] D. Curry, "Microsoft Teams Revenue and Usage Statistics (2021)," Business of Apps, 5 May 2021. [Online]. Available:

https://www.businessofapps.com/data/microsoftteams-statistics/. [Accessed 13 June 2021].

[3] P. W. Cardon and B. A. Marshall, "The hype and reality of social media use for work collaboration and team communication," International Journal of Business Communication, vol. 52, no. 3, pp. 273-293, 2015.

[4] A. McAfee, "Shattering the myths about Enterprise 2.0," Harvard Business Review, vol. 107, no. 2, pp. 235-245, 2009.

[5] D. Tapscott and A. D. Williams, Macrowikinomics: Rebooting business and the world, New York: Portfolio, 2010.

[6] E. Turban, T. P. Liang and S. P. Wu, "A framework for adopting collaboration 2.0 tools for virtual group decision making," Group Decisions and Negotiation, vol. 20, pp. 137-154, 2011.

[7] P. W. Cardon, "Community, culture, and affordances in social collaboration and communication," International Journal of Business Communication, vol. 53, no. 2, pp. 141-147, 2016.

[8] A. C. Fleischmann, J. Aritz and P. Cardon, "Language Proficiency and Media Synchronicity Theory: The Impact of Media Capabilities on Satisfaction and Inclusion in Multilingual Virtual Teams," in Proceedings of the 52nd Hawaii International Conference on System Science, Maui, HI, 2019.

[9] V. Garro-Abarca, P. Palos-Sanchez and M. AguayoCamacho, "Virtual Teams in Times of Pandemic: Factors That Influence Performance," Frontiers in Psychology, vol. 12, pp. 1-14, 2021.

[10] A. Anders, "Team communication platforms and emergent social collaboration practices,"
International Journal of Business Communication, vol. 53, no. 2, pp. 224-261, 2016.

[11] S. M. Ross, "Slack it to me: Complementing LMS with student-centric communications for the Millennial/Post-Millennial student," Journal of Marketing Education, vol. 41, no. 2, pp. 91-108, 2019.

[12] M. Haughey, "Collaborate with kindness: Consider these etiquette tips in Slack," Slack, 11 July 2019. [Online]. Available: https://slack.com/blog/collaboration/etiquette-tips-inslack. [Accessed 8 June 2021].

[13] V. Venkatesh, M. G. Morris, G. B. Davis and F. D. Davis, "User acceptance of information technology: Toward a unified view," MIS Quarterly, vol. 27, no. 3, pp. 425-478, 2003.

[14] V. Venkatesh, J. Y. Thong and X. Xu, "Consumer acceptance and use of information technology: Extending the Unified Theory of Acceptance and Use of Technology," MIS Quarterly, vol. 36, no. 1, pp. 157-178, 2012.

[15] K. Wagner, F. Nimmermann and H. Schramm-Klein, "Is It Human? The Role of Anthropomorphism as a Driver for the Successful Acceptance of Digital Voice Assistants," in Proceedings of the 52nd Hawaii International Conference on System Science, Maui, HI, 2019.

[16] S. Laumer, F. Gubler, C. Maier and T. Weitzel, "Job Seekers' Acceptance of Job Recommender Systems: Results of an Empirical Study," in Proceedings of the 51st Hawaii International Conference on System Science, Big Island, HI, 2018.

[17] G. Baptista and T. Oliveira, "Understanding mobile banking: The unified theory of acceptance and use of technology combined with cultural moderators," Computers in Human Behavior, vol. 50, no. Sep, pp. 418-430, 2015.

[18] F. D. Davis, R. P. Bagozzi, and P. R. Warshaw, "Extrinsic and intrinsic motivation to use computers in the workplace," Journal of Applied Social Psychology, vol. 22, no. 14, pp. 1111-1132, 1992.

[19] C.-M. Chao, "Factors Determining the Behavioral Intention to Use Mobile Learning: An Application and Extension of the UTAUT Model," Frontiers in Psychology, vol. 10, no. 1652, pp. 1-14, 2019.

[20] S. A. Brown, A. R. Dennis and V. Venkatesh, "Predicting Collaboration Technology Use: Integrating Technology Adoption and Collaboration Research," Journal of Management Information Systems, vol. 27, no. 2, pp. 9-53, 2010.

[21] A. Edmondson, "Psychological Safety and Learning Behavior in Work Teams," Administrative Science Quarterly, vol. 44, no. 4, pp. 350-383, 1999.

[22] Y. Zhang, Y. Fang, K.-K. Wei and H. Chen, "Exploring the role of psychological safety in promoting the intention to continue sharing knowledge in virtual communities," International 
Journal of Information Management, vol. 30, no. 5, pp. 425-436, 2010.

[23] A. Newman, R. Donohueb and N. Evab, "Pscyhological safety: A systematic review of the literature," Human Resource Management Review, vol. 27, pp. 521-535, 2017.

[24] C. Gibson and J. Gibbs, "Unpacking the concept of virtuality: the effects of geographic dispersion, electronic dependence, dynamic structure, and national diversity on team innovation," Administrative Science Quarterly, vol. 51, no. 3, p. 451-95, 2006.

[25] A. Ortega, M. Sánchez-Manzanares, Gil, F. and R. Rico, "Team Learning and Effectiveness in Virtual Project Teams: The Role of Beliefs about Interpersonal Context," The Spanish Journal of Psychology, vol. 13, no. 1, pp. 267-276, 2010.

[26] R. Dibble, L. Henderson and Z. Burns, "The Impact of Students' Cultural Intelligence on Their Psychological Safety in Global Virtual Project Teams," Journal of Teaching in International Business, vol. 30, no. 1, pp. 33-56, 2019.

[27] A. Edmondson and Z. Lei, "Psychological Safety: The History, Renaissance, and Future of an Interpersonal Construct," The Annual Review of Organizational Psychology, pp. 23-43, 2014.

[28] A. Lechner and J. T. Mortlock, "How to create psychological safety in virtual teams," Organizational Dynamics, vol. In press, 2021.

[29] R. Dibble, L. S. Henderson and Z. C. Burns, "The impact of students' cultural intelligence on their psychological safety in global virtual project teams," Journal of Teaching in International Business, vol. 30, no. 1, pp. 33-56, 2019.

[30] B. Kirkman, J. Cordery, J. Mathieu, B. Rosen and M. Kukenberger, "Global organizational communities of practice: The effects of nationality diversity, psychological safety, and media richness on community performance," Human Relations, vol. 66, no. 3, p. 333-362, 2013.

[31] J. Schepers, A. de Jong, M. Wetzels and K. de Ruyter, "Psychological safety and social support in groupware adoption: A multi-level assessment in education," Computers \& Eduction, vol. 51, pp. 757 775, 2008.

[32] H. Lee, "Changes in workplace practices during the COVID-19 pandemic: the roles of emotion, psychological safety and organisation support," Journal of Organizational Effectiveness: People and Performance, vol. 8, no. 1, pp. 97-128, 2021.

[33] B. L. Kirkman, J. L. Cordery, J. Mathieu, B. Rosen and M. Kukenberger, "Global organizational communities of practice: The effects of nationality diversity, psychological safety, and media richness on community performance," Human Relations, vol. 66, no. 3, pp. 333-362, 2013.
[34] V. Stray and N. B. Moe, "Understanding coordination in global software engineering: A mixed-methods study on the use of meetings and Slack," The Journal of Systems \& Software, vol. 170, pp. 1-20, 2020.

[35] M. Azarova, M. Hazoglou and E. Aronoff-Spencer, "Just slack it: A study of multidisciplinary teamwork based on ethnography and data from online collaborative software," New Media \& Society, vol. In Press, pp. 1-24, 2020.

[36] R. P. Bagozzi, "The Legacy of the Technology Acceptance Model and a Proposal for a Paradigm Shift," Journal of the Association for Information Systems, vol. 4, no. 244-254, p. 8, 2007.

[37] T. Barnett, A. W. Pearson, R. Pearson and F. W. Kellermanns, "Five-factor model personality traits as predictors of perceived and actual usage of technology," European Journal of Information Systems, vol. 24, no. 4, pp. 374-390, 2015.

[38] S. Devaraj, R. F. Easley and J. M. Grant, "How does personality matter? Relating the five-factor model to technology acceptance and use," Information Systems Research, vol. 19, no. 1, pp. 93-105, 2008.

[39] J. C. McElroy, A. R. Hendrickson, A. M. Townsend and S. M. Demarie, "Dispositional factors in internet use: Personality versus cognitive style," MIS Quarterly, vol. 31, no. 4, pp. 107-124, 2007.

[40] G. B. Svendsen, J. K. Johnsen, L. Almås-Sørensen and J. Vitters $\varnothing$, "Personality and technology acceptance: The influence of personality factors on the core constructs of the Technology Acceptance Model," Behaviour \& Information Technology, vol. 32, no. 4, pp. 323-334, 2013.

[41] K. Makwana and G. B. Dave, "Confirmatory factor analysis of Neris Type Explorer ${ }^{\circledR}$ scale - a tool for personality assessment," International Journal of Management, vol. 11, no. 9, pp. 257-265, 2020.

[42] "16 personalities -- Core theory," NERIS Analytics Limited, [Online]. Available: 16personalities.com. [Accessed 5 June 2021].

[43] V. Venkatesh and M. G. Morris, "Why don't men ever stop to ask directions? Gender, social influence, and their role in technology acceptance and usage behavior," MIS Quarterly, vol. 24, no. 1, pp. 115139, 2000.

[44] A. Padilla-Meléndez, A. R. del Aguila-Obra and A. Garrido-Moreno, "Perceived playfulness, gender differences and technology acceptance model in a blended learning scenario," Computers \& Education, vol. 63, pp. 306-317, 2013.

[45] P. W. Cardon, H. Ma and C. Fleischmann, "Recorded Business Meetings and AI Algorithmic Tools: Negotiating Privacy Concerns, Psychological Safety, and Control," International Journal of Business Communication, 2021.

[46] J. F. George, M. Gupta, G. Giordano, A. M. Mills, V. M. Tennant and C. C. Lewis, "The Effects of 
Communication Media and Culture on Deception Detection Accuracy," MIS Quarterly, vol. 42, no. 2, pp. 551-575, 2018.

[47] H. Tenzer and M. Pudelko, "Media choice in multilingual virtual teams," Journal of International Business Studies, vol. 47, no. 4, pp. 427-452, 2016.
[48] A. R. Dennis, R. M. Fuller and J. S. Valacich, "Media, tasks, and communication processes: A theory of media synchronicity," MIS Quarterly, vol. 32, no. 3, pp. 575-600., 2008.

\section{Appendix}

Table 2. Psychology safety in global virtual teams.

\begin{tabular}{lcccc}
\hline Item & $N$ & $M$ & $S D$ & \% Agree \\
\hline I felt emotionally invested in my team. & 1,390 & 4.26 & 1.64 & 45.5 \\
I felt strong belonging to my team. & 1,389 & 4.30 & 1.69 & 47.0 \\
People on this team sometimes rejected others for being different. (R) & 1,388 & 6.40 & 1.18 & 90.8 \\
I felt as if the team's problems were my own. & 1,388 & 3.93 & 1.84 & 40.3 \\
I was afraid of making mistakes. (R) & 1,389 & 4.64 & 1.80 & 54.9 \\
I felt at ease to speak up about problems and tough issues. & 1,386 & 4.91 & 1.62 & 64.8 \\
It was easy to ask for help from my team members. & 1,389 & 5.04 & 1.64 & 67.3 \\
I felt appreciated as a member of my team. & 1,386 & 5.11 & 1.60 & 68.1 \\
\hline
\end{tabular}

Note. (R) indicates reversed-coded item. \% Agree refers to the percentage of respondents who selected "somewhat agree," "agree," or "strongly agree" on the 7-point Likert scale.

\section{Table 3. Technology Acceptance of Slack.}

\begin{tabular}{lcccc}
\hline UTAUT2 Constructs and Items & $N$ & $M$ & $S D$ & \% Agree \\
\hline Performance expectancy & & & & \\
$\quad$ Slack was useful in this project. & 1,387 & 5.61 & 1.519 & 79.2 \\
$\quad$ Using Slack enabled us to accomplish tasks more quickly. & 1,385 & 5.26 & 1.634 & 69.8 \\
$\quad$ Using Slack increased our productivity. & 1,383 & 5.13 & 1.679 & 65.9 \\
$\quad$ Slack increased my team's chances of getting a better grade. & 1,385 & 4.97 & 1.72 & 60.4 \\
Effort expectancy & & & & \\
Slack was clear and understandable. & 1,382 & 5.47 & 1.545 & 77.5 \\
It was easy for me to become skillful at using Slack. & 1,384 & 5.59 & 1.444 & 79.5 \\
Slack was easy to use. & 1,382 & 5.66 & 1.469 & 80.7 \\
$\quad$ Learning to operate Slack was easy. & 1,381 & 5.64 & 1.449 & 80.2 \\
Hedonic motivation & & & & \\
$\quad$ Slack made the project more interesting. & 1,380 & 4.72 & 1.762 & 54.2 \\
$\quad$ Working with Slack was fun. & 1,380 & 4.61 & 1.739 & 49.7 \\
I liked working with Slack. & 1,380 & 5.05 & 1.678 & 64.2 \\
\hline
\end{tabular}

Note. \% Agree refers to the percentage of respondents who selected "somewhat agree," "agree," or "strongly agree" on the 7-point Likert scale. 